\title{
A REVIEW ON THE RADIOPROTECTIVE ACTIVITY OF ORGANOGERMANIUM AND ORGANOSILICON COMPOUNDS
}

\author{
Ghassoub Rima1 ${ }^{1}$ Jacques Satgé*1, Rodolphe Dagiral' \\ Claude Lion², Henri Sentenac-Roumanou ${ }^{3}$, \\ Marc Fatôme ${ }^{4}$, Vincent Roman ${ }^{4}$ and Jean-Denis Laval ${ }^{4}$
}

1 Laboratoire d'Hétérochimie Fondamentale et Appliquée, UPRES-A 5069 du CNRS, Université Paul Sabatier, 118, route de Narbonne, F-31062 Toulouse Cedex 4, France

2 Institut de Topologie et de Dynamique des Systèmes de l'Université de Paris VII, Associé au CNRS, 1, rue Guy de la Brosse, F-75005 Paris, France

3 Direction des Recherches, Etudes et Techniques, 26, boulevard Victor, F-00460 Armées, France

${ }^{4}$ Unité de Radioprotection, Centre de Recherche du Service de Santé des Armées, 24, avenue des Maquis du Grésivaudan, F-38702 La Tronche Cedex, France

\section{Abstract}

The present review describes the work carried out during the last 20 years in the field of the radioprotective activity and toxicity of several classes of organosilicon and organogermanium compounds (i.e. metallathiazolidines, metalladithioacetals, metallatranes and germathianes).

\section{Introduction}

During the 20-year period between 1978 and 1998 the Délégation Générale pour l'Armement and Département de Chimie Pharmacologie, France, sponsored a coordinated antiradiation drug development program. The objective of this program was to develop a drug or combination of drugs with organometallated compounds which could be taken by military personnel or other populations to protect them from the effects of ionizing radiations in a nuclear weapons attack, medical treatments or, in general, all radiation exposition.

During the research program approximately 562 compounds were chemically synthesized and tested in mice for their radioprotective properties.

The vast majority of these compounds were metallathiazolidines, metalladithioacetals of $\mathrm{N}$ substituted cysteamine, methylcysteamine, $N$-(2-thioethyl)-1,3-diaminopropane, $N$-substituted cysteamine or methylcysteamine by naphthylmethylimidazoline and $N$-substituted naphthylmethylimidazoline.

120 compounds of these derivatives are characterized by a dose reduction factor (DRF) between 1.3 and 1.75 .

The radioprotective activity and toxicity of several classes of organometallated derivatives (silathiazolidines, germathiazolidines, siladithioacetals, germadithioacetals, germatranes, silatranes and germylated sulfides) and their synthesis are reported.

All the biological tests have been performed in the Centre de Recherche du Service de Santé des Armées, La Tronche, France.

\section{Sila- and germathiazolidines}

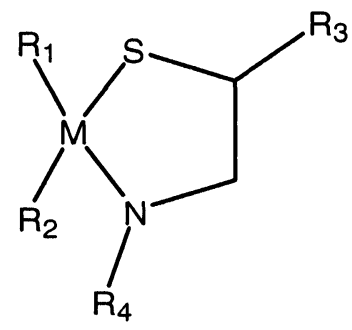

Sila- and germathiazolidines of $\mathrm{N}$-substituted cysteamine, methylcysteamine, $\mathrm{N}$-(2-thioethyl)1,3-diaminopropane and $N$-substituted cysteamine or methylcysteamine by naphthylmethylimidazoline were prepared according to two methods of heterocyclisation already described in the literature [1-3]. 


\section{Method A}

The action of diorganosilicon and -germanium dichloride [3] (in stoichiometric amounts) on $\mathrm{N}$ substituted cysteamine, methylcysteamine, $\mathrm{N}$-(2-thioethyl)-1,3-diaminopropane and $\mathrm{N}$-substituted cysteamine or methylcysteamine by naphthylmethylimidazoline in refluxing anhydrous tetrahydrofuran in the presence of freshly distilled triethylamine gave by a cyclisation reaction, with elimination of hydrochloric acid from $\mathrm{M}-\mathrm{Cl}$ and $\mathrm{SH}$ and $\mathrm{NH}$ [4] groups the corresponding products, Scheme 1.

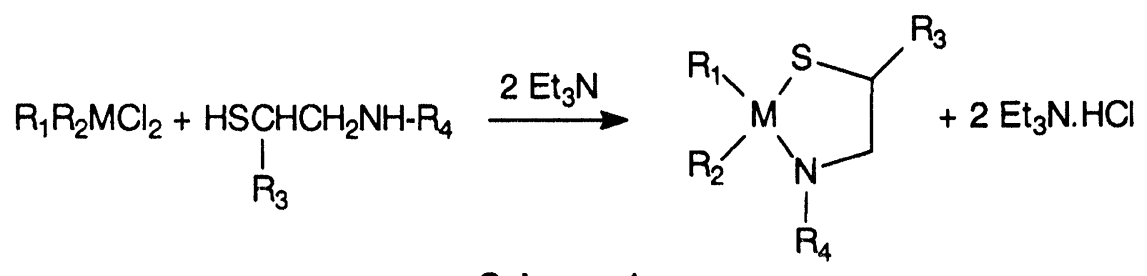

\section{Scheme 1}

\section{Method B}

The reaction of $\mathrm{N}$-substituted cysteamine, methylcysteamine, $\mathrm{N}$-(2-thioethyl)-1,3diaminopropane and $\mathrm{N}$-substituted cysteamine or methylcysteamine by naphthylmethylimidazoline, in stoichiometric amounts, with the bis(diethylamino)dialkylsilanes or -germanes in anhydrous tetrahydrofuran resulted in the cleavage of $\mathrm{M}-\mathrm{N}$ bonds by the $\mathrm{NH}$ and $\mathrm{SH}$ groups (a transamination reaction) [1, 3-5], forming the corresponding sila- and germathiazolidines in good yields, Scheme 2.

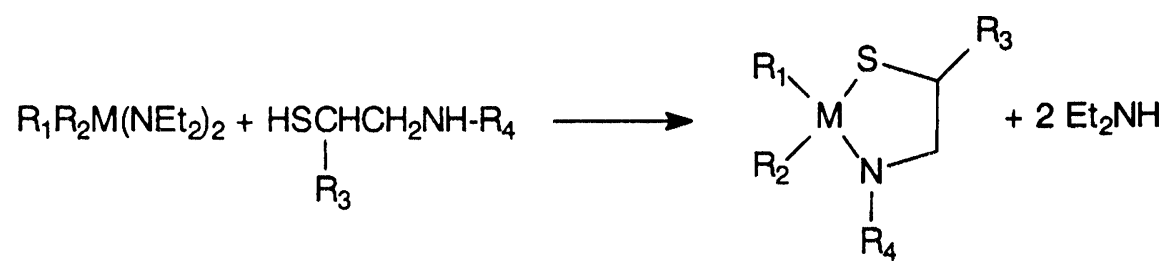

\section{Scheme 2}

A radioprotective study of metallathiazolidines shows that the silylated and germylated derivatives have a greater biological activity and a lower toxicity than that of the corresponding organic compounds [1] (Table I).

\section{Sila- and germadithioacetals}

\section{$\mathrm{R}_{1} \mathrm{R}_{2} \mathrm{M}\left[\mathrm{S}-\mathrm{CH}\left(\mathrm{R}_{3}\right)-\mathrm{CH}_{2} \mathrm{R}_{4}\right]_{2}$}

Sila- and germadithioacetals of $\mathrm{N}$-substituted cysteamine, methylcysteamine, $\mathrm{N}$-(2-thioethyl)1,3-diaminopropane, $\quad N$-substituted cysteamine and methylcysteamine by naphthylmethylimidazoline and $\mathrm{N}$-substituted naphthylmethylimidazoline were also obtained by two methods $C$ and $D[10]$.

\section{Method C}

The action of diorganosilicon and -germanium dichloride [3] on two equivalents of $\mathrm{N}$ substituted cysteamine, methylcysteamine, $\mathrm{N}$-(2-thioethyl)-1,3-diaminopropane, $\mathrm{N}$-substituted cysteamine and methylcysteamine by naphthylmethylimidazoline and $N$-substituted naphthylmethylimidazoline in refluxing anhydrous tetrahydrofuran in the presence of freshly distilled triethylamine gave the acyclic derivatives, Scheme 3.

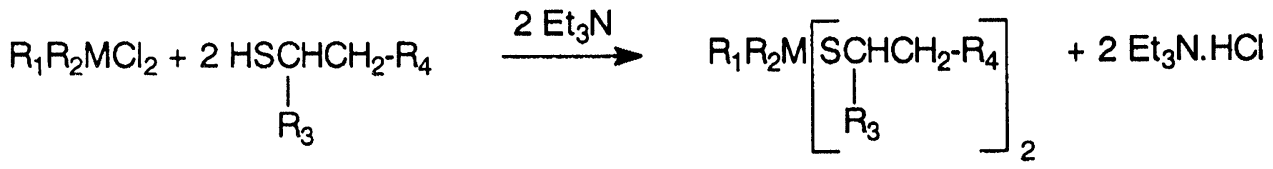

Scheme 3 
Table I - Radioprotective activity of some selected metallathiazolidines

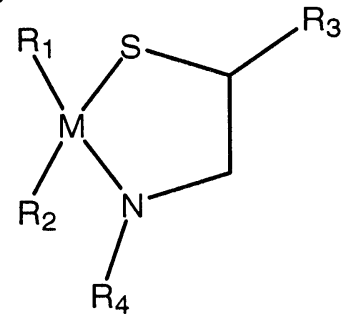

\begin{tabular}{|c|c|c|c|c|c|c|c|c|c|}
\hline \multicolumn{10}{|c|}{$M=S i$} \\
\hline $\begin{array}{l}\text { Cpd } \\
\text { [Ref.] }\end{array}$ & $\mathrm{R}_{1}$ & $\overline{R_{2}}$ & $\mathbf{R}_{3}$ & $\mathbf{R}_{4}$ & \begin{tabular}{|c|}
$\mathrm{LD}_{50}$ \\
$\left(\mathrm{mg} \cdot \mathrm{kg}^{-1}\right)$
\end{tabular} & $\begin{array}{c}\text { Injected } \\
\text { dose } \\
\left(\mathrm{mg} \cdot \mathrm{kg}^{-1}\right)\end{array}$ & $\begin{array}{c}\text { Irradiation } \\
\text { G y } \\
(t, \text { min })^{a} \\
\end{array}$ & $\begin{array}{c}\text { Survival } \\
\text { rate } \\
\% \\
\end{array}$ & DRF \\
\hline $\begin{array}{c}1 \\
{[1]}\end{array}$ & $\mathrm{CH}_{3}$ & $\mathrm{C}_{6} \mathrm{H}_{5}$ & $\mathrm{H}$ & H & 800 & $\begin{array}{l}400 \\
400 \\
400 \\
100\end{array}$ & $\begin{array}{c}10(15) \\
10(120) \\
12(15) \\
10(15)\end{array}$ & $\begin{array}{l}93 \\
90 \\
70 \\
16\end{array}$ & 1.6 \\
\hline $\begin{array}{c}2 \\
{[1]}\end{array}$ & $\mathrm{CH}_{3}$ & $\mathrm{C}_{6} \mathrm{H}_{5}$ & $\mathrm{CH}_{3}$ & $\mathrm{H}$ & 750 & \begin{tabular}{r|}
375 \\
375 \\
375 \\
93.7 \\
\end{tabular} & $\begin{array}{c}10(15) \\
10(120) \\
12(15) \\
10(15) \\
\end{array}$ & $\begin{array}{c}95 \\
0 \\
89 \\
75 \\
\end{array}$ & 1.5 \\
\hline $\begin{array}{c}3 \\
{[1]}\end{array}$ & $\mathrm{C}_{2} \mathrm{H}_{5}$ & $\mathrm{C}_{2} \mathrm{H}_{5}$ & $\mathrm{H}$ & $\mathrm{H}$ & 1000 & $\begin{array}{l}500 \\
500 \\
500 \\
\end{array}$ & $\begin{array}{c}9.5(15) \\
9.5(120) \\
11.5(15) \\
\end{array}$ & $\begin{array}{l}80 \\
90 \\
50 \\
\end{array}$ & 1.4 \\
\hline $\begin{array}{c}4 \\
{[1]}\end{array}$ & $\mathrm{CH}_{3}$ & $p-\mathrm{CH}_{3} \mathrm{C}_{6} \mathrm{H}_{4}$ & $\mathrm{H}$ & $\mathrm{H}$ & 1500 & $\begin{array}{l}750 \\
750 \\
750 \\
187 \\
\end{array}$ & $\begin{array}{c}9.5(15) \\
9.5(120) \\
11.5(15) \\
9.5(15)\end{array}$ & $\begin{array}{c}90 \\
70 \\
60 \\
0 \\
\end{array}$ & 1.4 \\
\hline $\begin{array}{c}5 \\
{[1]}\end{array}$ & $\mathrm{CH}_{3}$ & $p$ - $\mathrm{CH}_{3} \mathrm{OC}_{6} \mathrm{H}_{4}$ & $\mathrm{H}$ & $\mathrm{H}$ & 1500 & $\begin{array}{l}750 \\
750 \\
750 \\
187\end{array}$ & $\begin{array}{c}9.5(15) \\
9.5(120) \\
11.5(15) \\
9.5(15)\end{array}$ & $\begin{array}{l}95 \\
60 \\
50 \\
27\end{array}$ & 1.4 \\
\hline $\begin{array}{c}6 \\
{[6]}\end{array}$ & $\mathrm{HCl} \cdot \mathrm{H}_{2} \mathrm{~N}\left(\mathrm{CH}_{2}\right)_{2} \mathrm{~S}$ & $\mathrm{HCl} \cdot \mathrm{H}_{2} \mathrm{~N}\left(\mathrm{CH}_{2}\right)_{2} \mathrm{~S}$ & H & (c) & 600 & $\begin{array}{c}300 \\
300 \\
300 \\
75 \\
\end{array}$ & $\begin{array}{l}7.5(15) \\
7.5(90) \\
9.5(15) \\
7.5(15)\end{array}$ & $\begin{array}{c}100 \\
50 \\
90 \\
30 \\
\end{array}$ & 1.6 \\
\hline $\begin{array}{c}7 \\
{[7]}\end{array}$ & $n-\mathrm{C}_{6} \mathrm{H}_{13}$ & $n-\mathrm{C}_{6} \mathrm{H}_{13}$ & $\mathrm{H}$ & (d) & 250 & $\begin{array}{c}125 \\
125 \\
125 \\
31.25\end{array}$ & $\begin{array}{c}8(15) \\
8(90) \\
10(15) \\
8(15) \\
\end{array}$ & $\begin{array}{c}100 \\
80 \\
0 \\
0 \\
\end{array}$ & 1.3 \\
\hline $\begin{array}{c}8 \\
{[7]}\end{array}$ & $n-\mathrm{C}_{6} \mathrm{H}_{13}$ & $n-\mathrm{C}_{6} \mathrm{H}_{13}$ & $\mathrm{CH}_{3}$ & (d) & 220 & $\begin{array}{l}110 \\
110 \\
110 \\
27.5 \\
\end{array}$ & $\begin{array}{c}8(15) \\
8(90) \\
10(15) \\
8(15) \\
\end{array}$ & $\begin{array}{c}80 \\
90 \\
0 \\
50\end{array}$ & 1.3 \\
\hline
\end{tabular}

a: $t=$ time between administration of compound and irradiation

b: dose reduction factor $=\left(L D_{50(30}\right.$ days) treated/LD $/ D_{50(30 \text { days })}$ untreated $)$.

c: $\mathrm{R}_{4}=$<smiles>CN1CCOCC1</smiles><smiles>[CH]=[W]=[W]</smiles> 


\begin{tabular}{|c|c|c|c|c|c|c|c|c|c|}
\hline \multicolumn{10}{|c|}{$M=G e$} \\
\hline $\begin{array}{c}\text { Cpd } \\
\text { [Ref.] }\end{array}$ & $\overline{R_{1}}$ & $\mathbf{R}_{2}$ & $\mathbf{R}_{3}$ & $\overline{\mathbf{R}_{4}}$ & \begin{tabular}{l|}
$\mathrm{LD}_{50}$ \\
$\left(\mathrm{mg} \cdot \mathrm{kg}^{-1}\right)$
\end{tabular} & $\begin{array}{c}\text { Injected } \\
\text { dose } \\
\left(\mathrm{mg}^{\mathrm{k}} \mathrm{kg}^{-1}\right)\end{array}$ & $\begin{array}{c}\text { Irradiation } \\
\text { Gy } \\
(t, \min )^{a}\end{array}$ & $\begin{array}{c}\text { Survival } \\
\text { rate } \\
\%\end{array}$ & PRFb \\
\hline $\begin{array}{c}9 \\
{[1]}\end{array}$ & $\mathrm{CH}_{3}$ & $\mathrm{C}_{6} \mathrm{H}_{5}$ & H & $\mathrm{H}$ & 600 & $\begin{array}{c}300 \\
300 \\
300 \\
75 \\
\end{array}$ & $\begin{array}{c}9.5(15) \\
11.5(15) \\
13.5(15) \\
9.5(15) \\
\end{array}$ & $\begin{array}{c}100 \\
70 \\
40 \\
50 \\
\end{array}$ & 1.6 \\
\hline $\begin{array}{l}10 \\
{[1]}\end{array}$ & $\mathrm{CH}_{3}$ & $\mathrm{C}_{6} \mathrm{H}_{5}$ & $\mathrm{CH}_{3}$ & $\mathrm{H}$ & 500 & $\begin{array}{l}250 \\
250 \\
250 \\
250 \\
250 \\
62.5\end{array}$ & $\begin{array}{c}9.5(15) \\
9.5(120) \\
11.5(15) \\
13.5(15) \\
15.5(15) \\
9.5(15)\end{array}$ & $\begin{array}{c}80 \\
0 \\
70 \\
70 \\
0 \\
5\end{array}$ & 1.75 \\
\hline $\begin{array}{l}11 \\
{[1]}\end{array}$ & $\mathrm{CH}_{3}$ & $p-\mathrm{CH}_{3} \mathrm{OC}_{6} \mathrm{H}_{4}$ & H & $\mathrm{H}$ & 700 & $\begin{array}{l}350 \\
350 \\
350 \\
87.5 \\
\end{array}$ & $\begin{array}{c}9.5(15) \\
9.5(120) \\
11.5(15) \\
9.5(15)\end{array}$ & $\begin{array}{l}100 \\
20 \\
70 \\
13 \\
\end{array}$ & 1.45 \\
\hline $\begin{array}{l}12 \\
{[1]}\end{array}$ & $\mathrm{CH}_{3}$ & $p-\mathrm{CH}_{3} \mathrm{OC}_{6} \mathrm{H}_{4}$ & $\mathrm{CH}_{3}$ & $\mathrm{H}$ & 600 & $\begin{array}{c}300 \\
300 \\
300 \\
75 \\
\end{array}$ & $\begin{array}{c}9.5(15) \\
9.5(120) \\
11.5(15) \\
9.5(15) \\
\end{array}$ & $\begin{array}{l}95 \\
20 \\
70 \\
20 \\
\end{array}$ & 1.45 \\
\hline $\begin{array}{l}13 \\
{[1]}\end{array}$ & $\mathrm{C}_{6} \mathrm{H}_{5}$ & $\mathrm{C}_{6} \mathrm{H}_{5}$ & $\mathrm{H}$ & $\mathrm{H}$ & 400 & $\begin{array}{l}200 \\
200 \\
100\end{array}$ & $\begin{array}{c}9.5(15) \\
11.5(15) \\
9.5(15)\end{array}$ & $\begin{array}{l}90 \\
30 \\
70 \\
\end{array}$ & 1.4 \\
\hline $\begin{array}{l}14 \\
{[1]}\end{array}$ & $n-\mathrm{C}_{4} \mathrm{H}_{9}$ & $n-C_{4} \mathrm{H}_{9}$ & H & H & 700 & $\begin{array}{l}350 \\
350 \\
350 \\
\end{array}$ & $\begin{array}{c}9.5(15) \\
9.5(45) \\
11.5(15) \\
\end{array}$ & $\begin{array}{l}80 \\
30 \\
70 \\
\end{array}$ & 1.45 \\
\hline $\begin{array}{l}15 \\
{[1]}\end{array}$ & $n-C_{4} H_{9}$ & $n-C_{4} H_{9}$ & $\mathrm{CH}_{3}$ & $\mathrm{H}$ & 300 & $\begin{array}{l}150 \\
150 \\
150 \\
37.5 \\
\end{array}$ & $\begin{array}{c}9.5(15) \\
9.5(90) \\
11.5(15) \\
9.5(15) \\
\end{array}$ & $\begin{array}{c}100 \\
90 \\
30 \\
80 \\
\end{array}$ & 1.4 \\
\hline $\begin{array}{l}16 \\
{[1]}\end{array}$ & $n-C_{5} H_{11}$ & $n-C_{5} H_{11}$ & $\mathrm{H}$ & $\mathrm{H}$ & 1000 & $\begin{array}{l}500 \\
500 \\
500 \\
125 \\
\end{array}$ & $\begin{array}{c}9.5(15) \\
9.5(120) \\
11.5(15) \\
9.5(15)\end{array}$ & $\begin{array}{l}80 \\
10 \\
50 \\
20\end{array}$ & 1.4 \\
\hline $\begin{array}{l}17 \\
{[1]}\end{array}$ & $n-C_{5} H_{11}$ & $n-C_{5} H_{11}$ & $\mathrm{CH}_{3}$ & H & 700 & $\begin{array}{l}350 \\
350 \\
87.5 \\
\end{array}$ & $\begin{array}{c}9.5(15) \\
11.5(15) \\
9.5(15) \\
\end{array}$ & $\begin{array}{l}95 \\
60 \\
5 \\
\end{array}$ & 1.4 \\
\hline $\begin{array}{l}18 \\
{[1]}\end{array}$ & i-C $\mathrm{C}_{5} \mathrm{H}_{11}$ & $i-C_{5} H_{11}$ & $\mathrm{CH}_{3}$ & $\mathrm{H}$ & 800 & $\begin{array}{l}400 \\
400 \\
400 \\
400 \\
100\end{array}$ & $\begin{array}{c}9.5(15) \\
9.5(120) \\
11.5(15) \\
13.5(15) \\
9.5(15)\end{array}$ & $\begin{array}{c}94 \\
22 \\
70 \\
5 \\
0\end{array}$ & 1.45 \\
\hline $\begin{array}{l}19 \\
{[8]}\end{array}$ & $\mathrm{CH}_{3}$ & $\mathrm{C}_{6} \mathrm{H}_{5}$ & $\mathrm{CH}_{3}$ & $\mathrm{CH}_{3} \mathrm{CO}$ & 900 & $\begin{array}{c}450 \\
450 \\
450 \\
112.5 \\
\end{array}$ & $\begin{array}{c}9(15) \\
9(120) \\
11(15) \\
9(5) \\
\end{array}$ & $\begin{array}{l}93 \\
80 \\
10 \\
50 \\
\end{array}$ & 1.35 \\
\hline $\begin{array}{l}20 \\
{[8]}\end{array}$ & T-C5 $\mathrm{H}_{11}$ & $i-C_{5} \mathrm{H}_{11}$ & $\mathrm{CH}_{3}$ & $\mathrm{CH}_{3} \mathrm{CO}$ & 1000 & $\begin{array}{l}500 \\
500 \\
500 \\
125 \\
62.5 \\
\end{array}$ & $\begin{array}{c}8.5(15) \\
8.5(120) \\
10.5(15) \\
8.5(15) \\
9(15) \\
\end{array}$ & $\begin{array}{c}100 \\
90 \\
10 \\
80 \\
20 \\
\end{array}$ & 1.35 \\
\hline $\begin{array}{l}21 \\
{[9]}\end{array}$ & $i-C_{5} H_{11}$ & i-C $\mathrm{C}_{5} \mathrm{H}_{11}$ & H & (a) & 800 & $\begin{array}{l}800 \\
800 \\
\end{array}$ & $\begin{array}{l}7.75(15) \\
7.75(90)\end{array}$ & $\begin{array}{l}80 \\
80 \\
\end{array}$ & 1.3 \\
\hline
\end{tabular}




\begin{tabular}{|c|c|c|c|c|c|c|c|c|c|}
\hline $\begin{array}{l}222 \\
{[9]}\end{array}$ & $n-C_{6} H_{13}$ & $n-C_{6} H_{13}$ & H & (a) & 1500 & $\begin{array}{l}750 \\
750 \\
750 \\
187 \\
\end{array}$ & $\begin{array}{l}7.5(15) \\
7.5(90) \\
9.5(15) \\
7.5(15)\end{array}$ & $\begin{array}{c}100 \\
90 \\
0 \\
40 \\
\end{array}$ & 1.5 \\
\hline $\begin{array}{l}23 \\
{[6]}\end{array}$ & $\mathrm{CH}_{3}$ & $p-\mathrm{CH}_{3} \mathrm{C}_{6} \mathrm{H}_{4}$ & $\mathrm{H}$ & $\begin{array}{l}(\mathrm{CH})_{2} 2 \\
\mathrm{CONH}_{2}\end{array}$ & $>1500$ & $\begin{array}{l}1000 \\
1000 \\
1000\end{array}$ & $\begin{array}{c}8.25(15) \\
8.25(90) \\
10.30(15)\end{array}$ & $\begin{array}{l}70 \\
60 \\
20\end{array}$ & 1.4 \\
\hline $\begin{array}{l}24 \\
{[7]}\end{array}$ & $i-C_{5} H_{11}$ & i-C $\mathrm{C}_{5} \mathrm{H}_{11}$ & $\mathrm{H}$ & (b) & 80 & $\begin{array}{l}40 \\
40 \\
40 \\
40 \\
10 \\
\end{array}$ & $\begin{array}{c}8(15) \\
8(90) \\
10(15) \\
10(90) \\
8(15) \\
\end{array}$ & $\begin{array}{c}50 \\
80 \\
0 \\
20 \\
10 \\
\end{array}$ & 1.33 \\
\hline $\begin{array}{l}25 \\
{[9]}\end{array}$ & $n-\mathrm{C}_{6} \mathrm{H}_{13}$ & $n-\mathrm{C}_{6} \mathrm{H}_{13}$ & $\mathrm{CH}_{3}$ & $\mathrm{H}$ & 1500 & $\begin{array}{l}1000 \\
1000 \\
1000 \\
\end{array}$ & $\begin{array}{l}7.5(15) \\
7.5(90) \\
9.5(15) \\
\end{array}$ & $\begin{array}{l}80 \\
90 \\
80 \\
\end{array}$ & 1.5 \\
\hline $\begin{array}{l}26 \\
{[7]}\end{array}$ & T-C $\mathrm{C}_{5} \mathrm{H}_{11}$ & $i-C_{5} \mathrm{H}_{11}$ & $\mathrm{CH}_{3}$ & (b) & 150 & $\begin{array}{c}75 \\
75 \\
75 \\
75 \\
18.75 \\
\end{array}$ & $\begin{array}{c}8(15) \\
8(90) \\
10(15) \\
10(90) \\
8(15) \\
\end{array}$ & $\begin{array}{c}30 \\
90 \\
0 \\
0 \\
40 \\
\end{array}$ & 1.3 \\
\hline $\begin{array}{l}27 \\
{[7]}\end{array}$ & $n-C_{6} H_{13}$ & $n-C_{6} \mathrm{H}_{13}$ & $\mathrm{CH}_{3}$ & (b) & 200 & $\begin{array}{c}100 \\
100 \\
100 \\
25 \\
\end{array}$ & $\begin{array}{c}8(15) \\
8(90) \\
10(15) \\
8(15) \\
\end{array}$ & $\begin{array}{l}60 \\
80 \\
30 \\
40 \\
\end{array}$ & 1.3 \\
\hline
\end{tabular}

a: $\mathrm{R}_{4}=$<smiles>CN1CCOCC1</smiles><smiles></smiles>

\section{Method D}

The reaction of bis(diethylamino)dialkylsilanes and -germanes with two equivalents of $\mathrm{N}$ substituted cysteamine, methylcysteamine, $N$-(2-thioethyl)-1,3-diaminopropane, $N$-substituted cysteamine or methylcysteamine by naphthylmethylimidazoline and $N$-substituted naphthylmethylimidazoline in anhydrous tetrahydrofuran (a cleavage reaction of $\mathrm{M}-\mathrm{N}$ bonds by the $\mathrm{SH}$ group) gave the corresponding organometallated derivatives, Scheme 4.
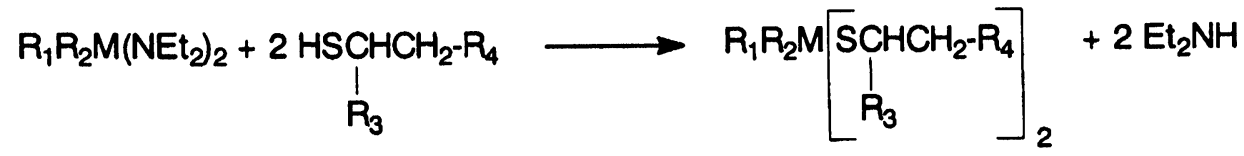

\section{Scheme 4}

\section{Sila- and germatranes}

$\mathrm{R}-\mathrm{M}\left(\mathrm{OCH}_{2} \mathrm{CH}_{2}\right)_{3} \mathrm{~N}$

Silatranes and germatranes have been synthesized according to two methods already known in the literature [13-18].

\section{Method E}

This method is the simplest and most general.

The reaction of trimethoxymetallanes, $\mathrm{R}-\mathrm{M}(\mathrm{OMe})_{3}(\mathrm{M}=\mathrm{Si}, \mathrm{Ge})$, obtained by the action of tetramethoxymetallanes [10] with cysteamine, methylcysteamine and $\mathrm{N}$-(2-thioethyl)-1,3diaminopropane in the presence of triethanolamine leads to corresponding metallatranes (Scheme 5). 
Table II - Radioprotective activity of some selected metalladithioacetals $\mathrm{R}_{1} \mathrm{R}_{2} \mathrm{M}\left[\mathrm{S}-\mathrm{CH}\left(\mathrm{R}_{3}\right)-\mathrm{CH}_{2} \mathrm{R}_{4}\right]_{2}$

\begin{tabular}{|c|c|c|c|c|c|c|c|c|c|}
\hline \multicolumn{10}{|c|}{$M=S i$} \\
\hline $\begin{array}{c}\text { Cpd } \\
{[R e f .]}\end{array}$ & $\overline{R_{1}}$ & $\overline{R_{2}}$ & $\overline{\mathbf{R}_{3}}$ & $\mathbf{R}_{\mathbf{4}}$ & $\begin{array}{c}\mathrm{LD}_{50} \\
\left(\mathrm{mg} \cdot \mathrm{kg}^{-1}\right)\end{array}$ & $\begin{array}{c}\text { Injected } \\
\text { dose } \\
\left(\mathrm{mg}^{\mathrm{kg}} \mathrm{kg}^{-1}\right)\end{array}$ & $\begin{array}{l}\text { Irradiation } \\
\text { Gy }(t, \min )^{a}\end{array}$ & $\begin{array}{c}\text { Survival } \\
\text { rate } \\
\%\end{array}$ & DRF' \\
\hline $\begin{array}{l}28 \\
{[11]}\end{array}$ & $\mathrm{CH}_{3}$ & $\mathrm{CH}_{3}$ & $\mathrm{H}$ & $\mathrm{NH}_{2}$ & 800 & $\begin{array}{l}400 \\
400 \\
400 \\
100\end{array}$ & $\begin{array}{c}9.5(15) \\
9.5(120) \\
11.5(40) \\
9.5(15)\end{array}$ & $\begin{array}{c}100 \\
90 \\
40 \\
33\end{array}$ & 1.4 \\
\hline $\begin{array}{l}29 \\
{[6]}\end{array}$ & $\mathrm{HCl} \cdot \mathrm{H}_{2} \mathrm{~N}\left(\mathrm{CH}_{2}\right)_{2} \mathrm{~S}$ & $\mathrm{HCl} \cdot \mathrm{H}_{2} \mathrm{~N}\left(\mathrm{CH}_{2}\right)_{2} \mathrm{~S}$ & $\mathrm{H}$ & (c) & 500 & $\begin{array}{l}250 \\
250 \\
250 \\
62.5\end{array}$ & $\begin{array}{l}7.5(15) \\
7.5(90) \\
9.5(15) \\
7.5(15)\end{array}$ & $\begin{array}{l}60 \\
10 \\
20 \\
30\end{array}$ & 1.3 \\
\hline $\begin{array}{c}30 \\
{[12]}\end{array}$ & i-C $\mathrm{C}_{5} \mathrm{H}_{11}$ & $i-C_{5} \mathrm{H}_{11}$ & $\mathrm{H}$ & (d) & 100 & $\begin{array}{c}50 \\
50 \\
50 \\
12.5\end{array}$ & $\begin{array}{l}7.75(15) \\
7.75(90) \\
9.75(15) \\
7.75(15)\end{array}$ & $\begin{array}{l}100 \\
80 \\
10 \\
60\end{array}$ & 1.4 \\
\hline $\begin{array}{c}31 \\
{[12]}\end{array}$ & $n-\mathrm{C}_{6} \mathrm{H}_{13}$ & $n-\mathrm{C}_{6} \mathrm{H}_{13}$ & H & (d) & 100 & $\begin{array}{c}50 \\
50 \\
50 \\
12.5\end{array}$ & $\begin{array}{l}7.75(15) \\
7.75(90) \\
9.75(15) \\
7.75(15)\end{array}$ & $\begin{array}{c}100 \\
100 \\
0 \\
50\end{array}$ & 1.5 \\
\hline
\end{tabular}

a: $t=$ time between administration of compound and irradiation.

b: dose reduction factor $=\left(L D_{50}(30\right.$ days $)$ treated/LD $/$ 50(30 days) untreated $)$.

c: $R_{4}=$

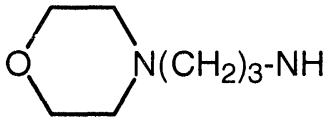

$\mathrm{d}: \mathrm{R}_{4}=$<smiles>c1ccc2ccccc2c1</smiles><smiles>CC1=NCCN1C</smiles>

\begin{tabular}{|c|c|c|c|c|c|c|c|c|c|}
\hline \multicolumn{10}{|c|}{$M=G e$} \\
\hline $\begin{array}{c}\text { Cpd } \\
\text { [Ref.] }\end{array}$ & $R_{1}$ & $\mathbf{R}_{2}$ & $\mathbf{R}_{3}$ & $\overline{\mathbf{R}_{4}}$ & \begin{tabular}{|c|}
$\mathrm{LD}_{50}$ \\
$\left(\mathrm{mg} \cdot \mathrm{kg}^{-1}\right)$
\end{tabular} & $\begin{array}{c}\text { Injected } \\
\text { dose } \\
\left(\mathrm{mg}^{\mathrm{kgg}} \mathrm{kg}^{-1}\right)\end{array}$ & $\begin{array}{c}\text { Irradiation } \\
\text { Gy } \\
(t, \text { min })^{a}\end{array}$ & $\begin{array}{c}\text { Survival } \\
\text { rate } \\
\%\end{array}$ & DRF \\
\hline $\begin{array}{l}32 \\
{[9]}\end{array}$ & (a) & (a) & $\mathrm{H}$ & $\mathrm{NH}_{2}$ & 500 & $\begin{array}{l}250 \\
250 \\
250 \\
62.5\end{array}$ & $\begin{array}{l}7.5(15) \\
7.5(90) \\
9.5(15) \\
7.5(15)\end{array}$ & $\begin{array}{c}80 \\
70 \\
50 \\
0\end{array}$ & 1.4 \\
\hline $\begin{array}{l}33 \\
{[11]}\end{array}$ & $n-\mathrm{C}_{4} \mathrm{H}_{9}$ & $n-\mathrm{C}_{4} \mathrm{H}_{9}$ & $\mathrm{H}$ & $\mathrm{NH}_{2}$ & 600 & $\begin{array}{l}300 \\
300\end{array}$ & $\begin{array}{c}9.5(15) \\
11.5(15)\end{array}$ & $\begin{array}{c}100 \\
10\end{array}$ & 1.3 \\
\hline $\begin{array}{c}34 \\
{[11]}\end{array}$ & $n-C_{4} H_{9}$ & $n-\mathrm{C}_{4} \mathrm{H}_{9}$ & $\mathrm{CH}_{3}$ & $\mathrm{NH}_{2}$ & 400 & $\begin{array}{c}200 \\
200 \\
200 \\
50\end{array}$ & $\begin{array}{c}9.5(15) \\
9.5(120) \\
11.5(15) \\
9.5(15)\end{array}$ & $\begin{array}{l}100 \\
90 \\
20 \\
70 \\
\end{array}$ & 1.3 \\
\hline $\begin{array}{l}35 \\
{[1}\end{array}$ & $1-\mathrm{C}_{5} \mathrm{H}_{11}$ & $i-\mathrm{C}_{5} \mathrm{H}_{11}$ & H & $\mathrm{NH}_{2}$ & 800 & $\begin{array}{l}400 \\
400 \\
400 \\
100\end{array}$ & $\begin{array}{c}9.5(15) \\
9.5(120) \\
11.5(15) \\
9.5(15)\end{array}$ & $\begin{array}{l}80 \\
50 \\
60 \\
30 \\
\end{array}$ & 1.4 \\
\hline $\begin{array}{c}36 \\
{[11]}\end{array}$ & $i-\mathrm{C}_{5} \mathrm{H}_{11}$ & $i-C_{5} \mathrm{H}_{11}$ & $\mathrm{CH}_{3}$ & $\mathrm{NH}_{2}$ & 600 & $\begin{array}{l}300 \\
300 \\
\end{array}$ & $\begin{array}{l}10(15) \\
12(15) \\
\end{array}$ & $\begin{array}{c}100 \\
95 \\
\end{array}$ & $>1.6$ \\
\hline $\begin{array}{c}37 \\
{[11]}\end{array}$ & $n-C_{5} H_{11}$ & $n-C_{5} H_{11}$ & $\mathrm{CH}_{3}$ & $\mathrm{NH}_{2}$ & 800 & $\begin{array}{l}400 \\
400 \\
400 \\
100\end{array}$ & $\begin{array}{c}9.5(15) \\
9.5(120) \\
11.5(15) \\
9.5(15)\end{array}$ & $\begin{array}{c}100 \\
50 \\
40 \\
40 \\
\end{array}$ & 1.4 \\
\hline
\end{tabular}




\begin{tabular}{|c|c|c|c|c|c|c|c|c|c|}
\hline $\begin{array}{c}38 \\
{[11]}\end{array}$ & $\mathrm{CH}_{3}$ & $\mathrm{C}_{6} \mathrm{H}_{5}$ & $\mathrm{CH}_{3}$ & $\mathrm{NH}_{2}$ & 600 & $\begin{array}{c}300 \\
300 \\
300 \\
75 \\
\end{array}$ & $\begin{array}{c}9.5(15) \\
9.5(120) \\
11.5(15) \\
9.5(15)\end{array}$ & $\begin{array}{l}90 \\
20 \\
90 \\
45 \\
\end{array}$ & $>1.5$ \\
\hline $\begin{array}{c}39 \\
{[11]}\end{array}$ & $\mathrm{CH}_{3}$ & $p-\mathrm{CH}_{3}-\mathrm{C}_{6} \mathrm{H}_{4}$ & $\mathrm{CH}_{3}$ & $\mathrm{NH}_{2}$ & 800 & $\begin{array}{l}400 \\
400 \\
400 \\
100\end{array}$ & $\begin{array}{c}9.5(15) \\
9.5(120) \\
11.5(15) \\
9.5(15)\end{array}$ & $\begin{array}{l}85 \\
70 \\
60 \\
33\end{array}$ & 1.5 \\
\hline $\begin{array}{l}40 \\
{[8]}\end{array}$ & $i-\mathrm{C}_{5} \mathrm{H}_{11}$ & $i-\mathrm{C}_{5} \mathrm{H}_{11}$ & $\mathrm{CH}_{3}$ & $\mathrm{NH}\left(\mathrm{CH}_{2}\right)_{3} \mathrm{NH}_{2}$ & 300 & $\begin{array}{l}150 \\
150 \\
150 \\
37.5\end{array}$ & $\begin{array}{c}9(15) \\
9(120) \\
11(15) \\
9(15)\end{array}$ & $\begin{array}{l}87 \\
40 \\
40 \\
90\end{array}$ & 1.4 \\
\hline $\begin{array}{l}41 \\
{[9]}\end{array}$ & $i-\mathrm{C}_{5} \mathrm{H}_{11}$ & $i-\mathrm{C}_{5} \mathrm{H}_{11}$ & $\mathrm{H}$ & (b) & 900 & $\begin{array}{c}450 \\
450 \\
450 \\
112.5\end{array}$ & $\begin{array}{l}7.75(15) \\
7.75(90) \\
9.75(15) \\
7.75(15)\end{array}$ & $\begin{array}{c}50 \\
80 \\
0 \\
50\end{array}$ & 1.3 \\
\hline $\begin{array}{l}42 \\
{[9]}\end{array}$ & $n-\mathrm{C}_{6} \mathrm{H}_{13}$ & $n-\mathrm{C}_{6} \mathrm{H}_{13}$ & $\mathrm{H}$ & (b) & 800 & $\begin{array}{l}400 \\
400 \\
400 \\
100 \\
\end{array}$ & $\begin{array}{l}7.5(15) \\
7.5(90) \\
9.5(15) \\
7.5(15)\end{array}$ & $\begin{array}{c}100 \\
80 \\
0 \\
50 \\
\end{array}$ & 1.5 \\
\hline $\begin{array}{l}43 \\
{[6]}\end{array}$ & $i-\mathrm{C}_{5} \mathrm{H}_{11}$ & $i-\mathrm{C}_{5} \mathrm{H}_{11}$ & H & $\mathrm{NH}\left(\mathrm{CH}_{2}\right)_{2} \mathrm{CONH}_{2}$ & $>1500$ & $\begin{array}{l}1000 \\
1000 \\
1000 \\
\end{array}$ & $\begin{array}{c}8.25(15) \\
8.25(90) \\
10.25(15) \\
\end{array}$ & $\begin{array}{c}100 \\
90 \\
30 \\
\end{array}$ & 1.4 \\
\hline $\begin{array}{l}44 \\
{[6]}\end{array}$ & $n-\mathrm{C}_{6} \mathrm{H}_{13}$ & $n-\mathrm{C}_{6} \mathrm{H}_{13}$ & $\mathrm{H}$ & $\mathrm{NH}\left(\mathrm{CH}_{2}\right)_{2} \mathrm{CONH}$ & $>1500$ & $\begin{array}{l}1000 \\
1000\end{array}$ & $\begin{array}{l}8.25(15) \\
8.25(90)\end{array}$ & $\begin{array}{l}90 \\
20\end{array}$ & 1.3 \\
\hline $\begin{array}{c}45 \\
{[12]}\end{array}$ & $i-\mathrm{C}_{5} \mathrm{H}_{11}$ & $i-\mathrm{C}_{5} \mathrm{H}_{11}$ & $\mathrm{H}$ & (c) & 80 & $\begin{array}{c}50 \\
50 \\
50 \\
50 \\
50 \\
50 \\
12.5\end{array}$ & $\begin{array}{c}7.75(15) \\
7.75(90) \\
7.75(180) \\
9.75(15) \\
9.75(90) \\
11.75(15) \\
7.75(15)\end{array}$ & $\begin{array}{c}100 \\
100 \\
90 \\
70 \\
0 \\
30 \\
80\end{array}$ & 1.7 \\
\hline $\begin{array}{c}46 \\
{[12]}\end{array}$ & $n-\mathrm{C}_{6} \mathrm{H}_{13}$ & $n-\mathrm{C}_{6} \mathrm{H}_{13}$ & $\mathrm{H}$ & (c) & 150 & $\begin{array}{c}75 \\
75 \\
75 \\
18.75 \\
\end{array}$ & $\begin{array}{l}7.75(15) \\
7.75(90) \\
9.75(15) \\
7.75(15)\end{array}$ & $\begin{array}{c}90 \\
100 \\
10 \\
70 \\
\end{array}$ & 1.4 \\
\hline $\begin{array}{l}47 \\
{[9]}\end{array}$ & $n-\mathrm{C}_{6} \mathrm{H}_{13}$ & $n-\mathrm{C}_{6} \mathrm{H}_{13}$ & $\mathrm{CH}_{3}$ & $\mathrm{NH}_{2}$ & 800 & $\begin{array}{l}800 \\
800 \\
800\end{array}$ & $\begin{array}{l}7.5(15) \\
7.5(90) \\
9.5(15)\end{array}$ & $\begin{array}{c}100 \\
70 \\
90\end{array}$ & $\geq 1.6$ \\
\hline $\begin{array}{l}48 \\
{[7]}\end{array}$ & $n-\mathrm{C}_{6} \mathrm{H}_{13}$ & $n-C_{6} \mathrm{H}_{13}$ & $\mathrm{CH}_{3}$ & (d) & 160 & $\begin{array}{l}80 \\
80 \\
80 \\
80 \\
20\end{array}$ & $\begin{array}{c}8(15) \\
8(90) \\
10(15) \\
10(90) \\
8(15)\end{array}$ & $\begin{array}{c}60 \\
100 \\
10 \\
0 \\
50\end{array}$ & 1.3 \\
\hline
\end{tabular}

$a: R_{1}=R_{2}=\|$

b: $\mathrm{R}_{4}=$<smiles>NCCN1CCOCC1</smiles>

c: $\mathrm{R}_{4}=$<smiles>CN1CCN=C1Cc1cccc2ccccc12</smiles>

$\mathrm{d}: \mathrm{R}_{4}=$<smiles>NCCN1CCN=C1Cc1cccc2ccccc12</smiles> 


$$
\mathrm{M}(\mathrm{OMe})_{4}+\mathrm{R}-\mathrm{H} \underset{-\mathrm{MeOH}}{\longrightarrow} \mathrm{RM}(\mathrm{OMe})_{3} \frac{\mathrm{N}\left(\mathrm{CH}_{2} \mathrm{CH}_{2} \mathrm{OH}\right)_{3}}{-3 \mathrm{MeOH}} \mathrm{R}-\mathrm{M}\left(\mathrm{OCH}_{2} \mathrm{CH}_{2}\right)_{3} \mathrm{~N}
$$

\section{Scheme 5}

\section{Method F}

The second method of synthesis of silatranes and germatranes includes of methoxymetallatranes. Thus, methoxymetallatranes obtained by the reaction of tetramethoxymetallanes with triethanolamine gave a condensation reaction with cysteamine, methylcysteamine and $\mathrm{N}$-(2-thioethyl)-1,3-diaminopropane affording the metallatranes (Scheme 6).

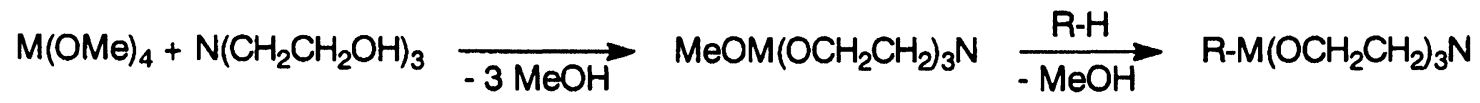

$$
\begin{aligned}
& M=\mathrm{Si}, \mathrm{Ge} ; \mathrm{R}=-\underset{R^{\prime}}{\mathrm{SCHCH}} \mathrm{H}_{2} \mathrm{NH}_{2} \cdot \mathrm{HCl},-\mathrm{S}\left(\mathrm{CH}_{2}\right)_{2} \mathrm{NH}\left(\mathrm{CH}_{2}\right)_{3} \mathrm{NH}_{2} \cdot \mathrm{HCl} ; \mathrm{R}^{\prime}=\mathrm{H}, \mathrm{CH}_{3}
\end{aligned}
$$

Scheme 6

Table III - Radioprotective activity of some selected metallatranes

\begin{tabular}{|c|c|c|c|c|c|c|c|c|}
\hline $\begin{array}{l}\text { Cppd } \\
\text { [Ref.] }\end{array}$ & $\bar{M}$ & $\overline{\mathbf{R}}$ & $\bar{Y}$ & $\frac{L^{\prime} D_{50}}{\left(\mathrm{mg} \mathrm{kg}^{-1}\right)}$ & $\begin{array}{l}\text { Injected } \\
\text { dose } \\
\left(\mathrm{mg}^{\mathrm{kgg}}{ }^{-1}\right)\end{array}$ & $\begin{array}{c}\text { Irradiation } \\
\text { Gy } \\
(t, \text { min })^{\mathbf{a}}\end{array}$ & $\begin{array}{c}\text { Survival } \\
\text { rate } \\
\%\end{array}$ & $\overline{\text { DRF }}$ \\
\hline $\begin{array}{c}49 \\
{[10]}\end{array}$ & $\mathrm{Si}$ & $\overline{\mathrm{H}}$ & $\mathrm{H.HCl}$ & 600 & $\begin{array}{c}300 \\
300 \\
75\end{array}$ & $\begin{array}{c}9.75(15) \\
11.75(15) \\
9.75(15)\end{array}$ & $\begin{array}{l}80 \\
10 \\
40\end{array}$ & 1.3 \\
\hline $\begin{array}{c}50 \\
{[10]}\end{array}$ & $\mathrm{Ge}$ & $\overline{\mathrm{H}}$ & $\mathrm{H.HCl}$ & 700 & $\begin{array}{r}350 \\
350 \\
350 \\
87.5 \\
\end{array}$ & $\begin{array}{c}9.75(15) \\
9.75(120) \\
11.75(15) \\
9.75(15)\end{array}$ & $\begin{array}{c}80 \\
0 \\
40 \\
0 \\
\end{array}$ & 1.4 \\
\hline $\begin{array}{c}51 \\
{[10]}\end{array}$ & $\mathrm{Ge}$ & $\bar{H}$ & $\mathrm{H}_{2} \mathrm{~N}\left(\mathrm{CH}_{2}\right)_{3}$ & 300 & $\begin{array}{l}150 \\
150 \\
150 \\
37.5 \\
\end{array}$ & $\begin{array}{c}9.5(15) \\
9.5(120) \\
11.5(15) \\
9.5(15)\end{array}$ & $\begin{array}{c}60 \\
0 \\
40 \\
10 \\
\end{array}$ & $\overline{1.4}$ \\
\hline $\begin{array}{c}52 \\
{[10]}\end{array}$ & $\mathrm{Ge}$ & $\overline{\mathrm{H}}$ & $2 \mathrm{HCl}_{2} \mathrm{H}_{2} \mathrm{~N}\left(\mathrm{CH}_{2}\right)_{3}$ & 900 & $\begin{array}{c}450 \\
450 \\
450 \\
112.5\end{array}$ & $\begin{array}{c}9.5(15) \\
9.5(120) \\
11.5(15) \\
9.5(15)\end{array}$ & $\begin{array}{l}100 \\
20 \\
30 \\
70\end{array}$ & 1.45 \\
\hline $\begin{array}{c}53 \\
{[10]}\end{array}$ & $\mathrm{Ge}$ & $\overline{\mathrm{CH}_{3}}$ & $\mathrm{H.HCl}$ & 1500 & $\begin{array}{l}1000 \\
1000 \\
1000\end{array}$ & $\begin{array}{c}10(15) \\
10(120) \\
12(15)\end{array}$ & $\begin{array}{c}80 \\
0 \\
70\end{array}$ & 1.5 \\
\hline
\end{tabular}
$\mathrm{YHN}-\mathrm{CH}_{2}-\mathrm{CH}(\mathrm{R})-\mathrm{SM}\left(\mathrm{OCH}_{2} \mathrm{CH}_{2}\right)_{3} \mathrm{~N}$

\section{Germylated sulfides}

$\left\{\left[\mathrm{HCl} . \mathrm{H}_{2} \mathrm{NCH}_{2}-\mathrm{CH}(\mathrm{R})-\mathrm{S}\right]_{2} \mathrm{GeS}\right\}_{3}$

\begin{tabular}{|c|c|c|c|c|c|c|}
\hline $\begin{array}{c}\text { Cpd } \\
\text { [Ref.] }\end{array}$ & $\mathbf{R}$ & $\begin{array}{c}\mathrm{LD}_{50} \\
\left(\mathrm{mg} \cdot \mathrm{kg}^{-1}\right)\end{array}$ & $\begin{array}{c}\text { Injected } \\
\text { dose } \\
\left(\mathrm{mg} \cdot \mathrm{kg}^{-1}\right)\end{array}$ & $\begin{array}{l}\text { Irradiation } \\
\text { Gy }(t, \min )^{a}\end{array}$ & $\begin{array}{c}\text { Survival rate } \\
\%\end{array}$ & DRF $^{b}$ \\
\hline $\begin{array}{l}54 \\
{[9]}\end{array}$ & $\mathrm{CH}_{3}$ & 1000 & $\begin{array}{l}500 \\
500 \\
500 \\
125\end{array}$ & $\begin{array}{l}7.5(15) \\
7.5(90) \\
9.5(15) \\
7.5(15)\end{array}$ & $\begin{array}{c}100 \\
50 \\
80 \\
10\end{array}$ & 1.6 \\
\hline $\begin{array}{c}55 \\
{[10]}\end{array}$ & $\overline{\mathrm{H}}$ & 800 & $\begin{array}{l}800 \\
800\end{array}$ & $\begin{array}{c}9.5(15) \\
11.5(15)\end{array}$ & $\begin{array}{c}100 \\
90\end{array}$ & $\geq 1.5$ \\
\hline
\end{tabular}

Table IV - Radioprotective activity of germylated sulfides 
The syntheses of these compounds were realized by the action of $\mathrm{NaSH}$ on $\left\{\left[\mathrm{HCl} . \mathrm{H}_{2} \mathrm{NCH}_{2} \mathrm{CH}(\mathrm{R})\right]_{2} \mathrm{SGeCl}_{2}\left(\mathrm{R}=\mathrm{H}, \mathrm{CH}_{3}\right)[10]\right.$ in anhydrous pyridine, Scheme 7.

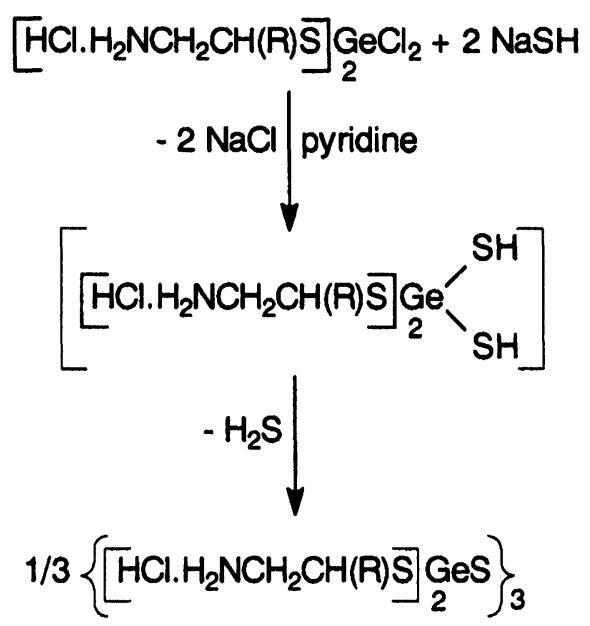

\section{Diisoamyldithiagermocane}

\section{Scheme 7}

\section{$\left(i-\mathrm{C}_{5} \mathrm{H}_{12}\right)_{2} \mathrm{Ge}\left(\mathrm{SCH}_{2} \mathrm{CH}_{2}\right)_{2} \mathrm{~S}$}

This compound was obtained by the reaction of 2,2'-thiodiethanethiol with diisoamylgermyldichloride [3], in equimolar amount, in anhydrous tetrahydrofuran and in the presence of freshly distilled triethylamine, Scheme 8.

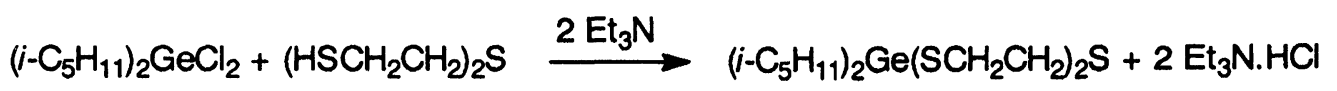

Scheme 8

Table V - Radioprotective activity of diisoamyldithiagermocane

\begin{tabular}{|c|c|c|c|c|c|}
\hline $\begin{array}{c}\text { Cpd } \\
\text { [Ref.] }\end{array}$ & $\begin{array}{c}\text { LD }_{\text {50 }} \\
\left(\mathbf{m g}^{\mathbf{k}} \mathbf{k g}^{-1}\right)\end{array}$ & $\begin{array}{c}\text { Injected } \\
\text { dose } \\
\left(\mathbf{m g}_{\mathbf{k g}} \mathbf{- 1}\right)\end{array}$ & $\begin{array}{c}\text { Irradiation } \\
\text { Gy (t, min) }\end{array}$ & $\begin{array}{c}\text { Survival rate } \\
\%\end{array}$ & DRFb \\
\hline $\mathbf{5 6}$ & $>1500$ & 1000 & $7.5(15)$ & 60 & 1.3 \\
{$[6]$} & & 1000 & $7.5(90)$ & 50 & \\
\hline
\end{tabular}

\section{7- Di-n-hexylpyridinooxathiagermolane}

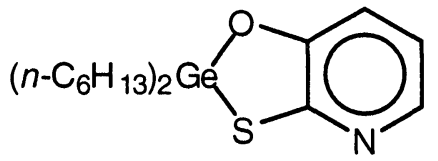

This product was prepared by action of dichlorodi-n-hexylgermanium [3] on 2-thio-3-pyridinol in refluxing anhydrous tetrahydrofuran in the presence of freshly distilled triethylamine, Scheme 9.

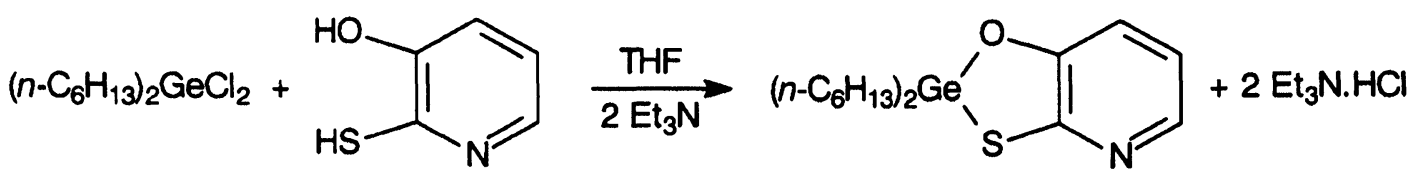

\section{Scheme 9}

\section{Pharmacology: evaluation of radioprotection}

Male CD1 mice (Charles River, France), $25 \mathrm{~g}$ body weight, were used. The compounds were injected intraperitoneally $15,90,120$ or $180 \mathrm{~min}$ before irradiation. The irradiation dose was $\mathrm{LD}_{100} / 30$ days for untreated mice $(7.5,7.75,8,8.25$ or $8.3 \mathrm{~Gy}$, according to the irradiation date) or a 
2 Gy greater dose. When necessary, other irradiation doses (between 8.5-13.5 Gy were tested in order to evaluate the irradiation $\mathrm{LD}_{50} / 30$ days of protected mice. The injected dose of the compound was equal to one-half or one-eighth of the $L D_{50}$ value which had been determined previously. The radioprotective effect was evaluated by the Dose Reduction Factor (DRF), which is the ratio between the $\mathrm{LD}_{50} / 30$ days of treated mice and that of control mice (between 6.5 and 6.75 Gy, according to the date).

Table VI - Radioprotective activity of di-n-hexylpyridinooxathiagermolane

\begin{tabular}{|c|c|c|c|c|c|}
\hline $\begin{array}{c}\text { Cpd } \\
\text { [Ref.] }\end{array}$ & $\begin{array}{c}\mathrm{LD}_{50} \\
\left(\mathrm{mg} \cdot \mathrm{kg}^{-1}\right)\end{array}$ & $\begin{array}{c}\text { Injected } \\
\text { dose } \\
\text { (mg.kg-1) }\end{array}$ & $\begin{array}{l}\text { Irradiation } \\
\text { Gy }(t, \min )^{a}\end{array}$ & $\begin{array}{c}\text { Survival rate } \\
\%\end{array}$ & DRF \\
\hline $\begin{array}{l}57 \\
{[6]}\end{array}$ & 800 & $\begin{array}{l}400 \\
400 \\
400 \\
100\end{array}$ & $\begin{array}{l}7.5(15) \\
7.5(90) \\
9.5(15) \\
7.5(15)\end{array}$ & $\begin{array}{c}40 \\
50 \\
0 \\
20\end{array}$ & 1.3 \\
\hline
\end{tabular}

Irradiation was applied using a cobalt-60 source at the dose rate of 0.3-0.4 Gy. $\min ^{-1}$ according to the date. During irradiation, animals were placed in a Plexiglass box with 30 cells in a homogeneous field, $28.5 \times 28.5 \mathrm{~cm}$ in area. Dosimetry was checked with an ionisation chamber dosimeter. The different $L_{50}$ values were determined by probit analysis.

\section{Conclusion}

The objective of this work was to incorporate potentially radioprotective organic groups in organometallic structures such as metallathiazolidines, metalladithioacetals, metallatranes and germathianes so as to decrease their toxicity and increase their radioprotective activity.

Tables I through VI summarize the radiation protection obtained in mice after intraperitoneal administration in miglyol solution of the organosilylated and organogermylated derivatives described. Generally, these organometallic compounds have a lower toxicity and a radioprotective activity equal to or greater than that of the starting organic derivatives (namely cysteamine [19], methylcysteamine [10] and $\mathrm{N}$-substituted cysteamine or methylcysteamine [6,9] and $\mathrm{N}$-substituted naphthylmethylimidazoline $[7,12])$. We have to underline that in some cases this great radioprotective activity was obtained with organosilylated or organogermylated derivatives injected in lower doses, expressed in mmol fraction, than those used for starting organic compounds.

The analysis of the toxicity and powerful radioprotective effect of all compounds presented in Table I shows that the metallathiazolidines have generally a good radioprotective activity and lower toxicity compared with starting organic derivatives.

Several compounds in this series $(\mathbf{1}, \mathbf{2}, \mathbf{6}, 9$ and 10) exhibit a dose reduction factor (DRF) of $1.5-1.75$.

The $\mathrm{LD}_{50}$ dose of each compound indicated that they were weakly toxic $\left(500-1500 \mathrm{mg} \cdot \mathrm{kg}^{-1}\right)$.

-Derivative $1\left(\mathrm{DRF}=1.6 ; \mathrm{LD}_{50}=800 \mathrm{mg} \cdot \mathrm{kg}^{-1}\right.$ ) at one-half of $L D_{50}$ (the maximum tolerated dose, MTD) this product protects $93 \%$ and $90 \%$ of mice at 10 Gy 15 or 120 minutes after injection and $70 \%$ survival at 12 Gy 15 minutes before irradiation. At one-eighth of the $L_{50}$, there was a 16 $\%$ survival at a dose of $10 \mathrm{~Gy}$.

-Derivative $2\left(D R F=1.5 ; L D_{50}=750 \mathrm{mg} \cdot \mathrm{kg}^{-1}\right)$ at one-half of $L_{50} 95 \%$ and $89 \%$ protection were obtained at doses of 10 and 12 Gy. At one-eighth of the LD $D_{50}$, there was still $75 \%$ survival at a dose of 10 Gy.

-Derivative $6\left(D R F=1.6 ; D_{50}=600 \mathrm{mg} \cdot \mathrm{kg}^{-1}\right)$ at one-half of $L D_{50} 100 \%$ and $90 \%$ survival were obtained at doses of 7.5 and $9.5 \mathrm{~Gy}$ and $50 \%$ survival was observed at a dose of $7.5 \mathrm{~Gy}$. At one-eighth of the $L D_{50}$, there was a $30 \%$ survival at a dose of 7.5 Gy.

-Derivative $9\left(\mathrm{DRF}=1.6 ; \mathrm{LD}_{50}=600 \mathrm{mg} \cdot \mathrm{kg}^{-1}\right)$ at one-half of $L_{50} 100 \%, 70 \%$ and $40 \%$ survival were obtained at doses of $9.5,11.5$ and 13.5 Gy. At one-eighth of the $L D_{50}$, there was a 50 $\%$ survival at a dose of 9.5 Gy.

-Derivative $10\left(D R F=1.75 ; L_{50}=500 \mathrm{mg}^{-\mathrm{kg}^{-1}}\right.$ ) at one-half of $L_{50} 80 \%, 70 \%$ and $70 \%$ survival were obtained at doses of $9.5,11.5$ and 13.5 Gy. 
The analysis of the results reported in Table II shows that the siladithioacetals and germadithioacetals $31,36,38,39,42,45$ and 47 have an important radioprotective activity (DRF between 1.5-1.7).

-Derivative $31\left(\mathrm{DRF}=1.5 ; \mathrm{LD}_{50}=100 \mathrm{mg} \cdot \mathrm{kg}^{-1}\right.$ ) at one-half of $L D_{50} 100 \%$ protection was observed at a dose of $7.75 \mathrm{~Gy}$. At one-eighth of the $\mathrm{LD}_{50}$, there was still $50 \%$ survival at a dose of 7.75 Gy.

-Derivative 36 (DRF>1.6; $L D_{50}=600 \mathrm{mg} \cdot \mathrm{kg}^{-1}$ ) at one-half of $L D_{50} 100 \%$ and $95 \%$ survival were obtained at doses of 10 and $12 \mathrm{~Gy}$.

-Derivative $38\left(D R F>1.5 ; L D_{50}=600 \mathrm{mg} \mathrm{kg}^{-1}\right)$ at one-half of $L D_{50} 90 \%$ survival was observed at doses of 9.5 or $11.5 \mathrm{~Gy}$. At one-eighth of the $\mathrm{LD}_{50}$, there was $45 \%$ survival at a dose of $9.5 \mathrm{~Gy}$.

-Derivative $39\left(\mathrm{DRF}=1.5 ; \mathrm{LD}_{50}=800 \mathrm{mg} \cdot \mathrm{kg}^{-1}\right)$ at one-half of $\mathrm{LD}_{50} 85 \%, 70 \%$ and $60 \%$ survival were obtained at doses of 9.5 and $11.5 \mathrm{~Gy}$. At one-eighth of the $\mathrm{LD}_{50}$, there was $33 \%$ survival at a dose of 9.5 Gy.

-Derivative 42 (DRF $=1.5 ; L D_{50}=800 \mathrm{mg} \cdot \mathrm{kg}^{-1}$ ) at one-half of $L D_{50} 100 \%$ and $80 \%$ survival were observed at a dose of 7.5 Gy. At one-eighth of the $L D_{50}$, there was still $50 \%$ survival at a dose of 7.5 Gy.

-Derivative 45 (DRF $=1.7 ; L_{50}=80 \mathrm{mg} \cdot \mathrm{kg}^{-1}$ ) at $L D_{50} / 1.6100 \%$ and $90 \%$ survival were observed at a dose of 7.75 Gy. $70 \%$ and $30 \%$ survival were also obtained at doses of 9.75 and 11.75 Gy. At $L D_{50} / 6.4$, there was still $80 \%$ survival at dose of 7.75 Gy.

-Derivative $47\left(\mathrm{DRF}>1.6 ; \mathrm{LD}_{50}=800 \mathrm{mg} \cdot \mathrm{kg}^{-1}\right)$ the administration of this product at of $L_{50}$ protects $100 \%$ and $70 \%$ of mice at a dose of 7.5 Gy and $90 \%$ survival was obtained at a dose of 9.5 Gy.

At last, it is important to note the significant radioprotective activity of organogermylated sulfides 54 and 55 (DRF $=1.5-1.6)$.

-Derivative $54\left(\right.$ DRF $=1.6 ; \mathrm{LD}_{50}=1000 \mathrm{mg} \cdot \mathrm{kg}^{-1}$ ) at the MTD this product protects $100 \%$ and $80 \%$ of mice at doses of 7.5 and $9.5 \mathrm{~Gy}$, it also protects $50 \%$ of mice at a dose of $7.5 \mathrm{~Gy}$.

-Derivative $55\left(\operatorname{DRF}\left(1.5 ; L_{50}=800 \mathrm{mg}^{\mathrm{kg}}{ }^{-1}\right)\right.$ at of $L D_{50} 100 \%$ and $90 \%$ protection were observed at doses of 9.5 and 11.5 Gy.

In short, several organosilylated and organogermylated compounds present a good radioprotective activity compared with the starting organic derivatives due to the presence in these molecules of organometallic groups which increase the hydrosolubility and by the presence of organic ligands which increase the liposolubility and biological activity of these molecules, thereby favouring their passage through the cellular membranes.

The results presented in this paper confirm the positive contribution of germanium and silicon in this field in agreement with previous works [1, 6-12, 20-22] and the interesting biological activity of organosilicon and organogermanium compounds in different fields [23-44].

\section{Acknowledgements}

The authors wish to thank the Délégation Générale pour l'Armement (D.G.A.), Département de Chimie-Pharmacologie, Ministère de la Défense Nationale, France, for their financial support and interest in this research.

\section{References}

1 J. Satgé, A. Cazes, M. Bouchaut, M. Fatôme, H. Sentenac-Roumanou and C. Lion, Eur. J. Med. Chem., 17 (1982) 433.

2 G. Dousse, J. Satgé and M. Rivière-Baudet, Synth. React. Inorg. Met.-Org. Chem., 3 (1973) 11.

3 M. Lesbre, P. Mazerolles and J. Satgé in: The Organic Compounds of Germanium, John Wiley and Sons, New York, (1973).

4 J. Satgé, M. Lesbre and M. Baudet, C. R. Acad. Sci. Paris. Ser. C. 259 (1964) 4733.

5 J. Satgé and M. Baudet, C. R. Acad. Sci. Paris. Ser. C. 263 (1966) 435.

6 G. Rima, J. Satgé, H. Sentenac-Roumanou, M. Fatôme, J. D. Laval, C. Lion, C. Thiriot, R. Dagiral and C. Martin, Main Group Met. Chem., 20 (1997) 255.

7 G. Rima, J. Satgé, R. Dagiral, C. Lion, M. Fatôme, V. Roman and J.-D. Laval, Appl. Organomet. Chem., (1999) (under press).

8 M. Fatôme, H. Sentenac-Roumanou, C. Lion, J. Satgé and G. Rima, Eur. J. Med. Chem., 23 (1988) 257.

9 G. Rima, J. Satgé, H. Sentenac-Roumanou, M. Fatôme, J. D. Laval, C. Lion and R. Dagiral, Appl. Organomet. Chem., 10 (1996) 113. 
10 J. Satgé, G. Rima, M. Fatôme, H. Sentenac-Roumanou and C. Lion, Eur. J. Med. Chem., 24 (1989) 48.

11 M. Fatôme, H. Sentenac-Roumanou, C. Lion, J. Satgé, M. Fourtinon and G. Rima, Eur. J. Med. Chem., 19 (1984) 119.

12 G. Rima, J. Satgé, R. Dagiral, C. Lion, M. Fatôme, V. Roman and J.-D. Laval, Metal-Based Drugs, 5 (1998) 139.

13 M. G. Voronkov and V. P. Baryshok, J. Organomet. Chem., 239 (1982) 199.

14 N. Yu. Khromova, T. K. Gar and V. F. Mironov in: Heteroorganic Compounds and their applications: Review of Germatranes and their Analogs, Niitekhim, (1985), Moscow.

15 M. G. Voronkov, Top. Curr. Chem., 84 (1979) 77.

16 M. G. Voronkov, Pure Appl. Chem., 19 (1969) 399.

17 R. Tacke and U. Wannagat, Top. Curr. Chem., 84 (1979) 1.

18 M. G. Voronkov, G. I. Zelchan and E. Lukevics, in Silicon and Life, Berlin Akad., Verlag, (1975).

19 L. Breuil, La Radioprotection chimique, Direction des Recherches, Etudes et Techniques, Paris Armées (1979).

20 G. Rima, J. Satgé, M. Fatôme, J. D. Laval, H. Sentenac-Roumanou, C. Lion and M. Lazraq, Eur. J. Med. Chem., 26 (1991) 291.

21 G. Rima, J. Satgé, H. Sentenac-Roumanou, M. Fatôme, C. Lion and J. D. Laval, Eur. J. Med. Chem., 28 (1993) 761.

22 G. Rima, J. Satgé, H. Sentenac-Roumanou, M. Fatôme, J. D. Laval, C. Lion, O. Alazard and P. Chabertier, Appl. Organomet. Chem., 8 (1994) 481.

$23 \mathrm{~J}$. Satgé, Propriétés et applications biologiques de dérivés organométalliques du silicium, du germanium et de l'étain, rapport de mise au point A. E. P. A., (juin 1981).

24 T. K. Gar and V. F. Mironov, in Review of the Biological Activity of Germanium Compounds, Niitekhim (eds), Moscow, (1982).

25 H. A. Meinema, A. M. J. Liebregts, H. A. Budding and E. J. Bulten, Rev. Silicon Germanium Tin Lead Compd., 8 (1985) 157.

26 G. Atassi, Rev. Silicon Germanium Tin Lead Compd., 8 (1985) 219.

27 N. Kakimoto, M. Matsui, T. Takada and M. Akiba, Heterocycles, 23 (1985) 2681.

28 T. K. Miyamoto, N. Sugita, Y. Matsumoto, Y. Sasaki and H. Bunkyoku, Chem. Lett., (1983) 1695.

29 J. S. Thayer, Appl. Organometal. Chem., 1(1987)227; Rev. Silicon, Germanium Tin Lead Compd., 8 (1985) 133.

30 R. Tacke, B. Becker, D. Berg, W. Brandes, S. Dutzmann and K. Schaller, J. Organomet. Chem., 438 (1992) 45.

31 E. Lukevics and L. Ignatovich, Appl. Organomet. Chem., 6 (1992) 113.

32 E. Lukevics, L. Ignatovich, N. Shilina and S. Germane, Appl. Organomet. Chem., 6 (1992) 261.

33 E. Lukevics, S. Germane and L. Ignatovich, Appl. Organomet. Chem., 6 (1992) 543.

34 F. Anger, J. P. Anger, L. Guillou and A. Papillon, Appl. Organomet. Chem., 6 (1992) 267.

35 R. Tacke, D. Reichel, P. G. Jones, X. Hou, M. Waelbroeck, J. Gross, E. Mutschler and G. Lambrecht, J. Organomet. Chem., 521 (1996) 305.

36 E. Lukevics, V. Vevenis and V. Dirnens, Appl. Organomet. Chem., 11 (1997) 805.

37 E. Lukevics, L. Ignatovich and S. Germane, Khim. Geterotsikl. Soed., 10 (1995) 1412.

38 E. Lukevics and L. Ignatovich, Main Group Met. Chem., 1 (1994) 133

39 E. Lukevics, T. K. Gar, L. Ignatovich and V. F. Mironov, Biological activity of the germanium containing compounds, Riga, "Zinatne" (1990). (in Russian).

40 F. Li, Z. Zhang, and H. Gao, Metal Based Drugs, 5 (1996) 241.

41 E. Lukevics, S. Germane, A. Zidermane, A. Dauvarte, I. M. Kravchenko, M. Trusule, V. F. Mironov, T. K. Gar et al., Khim. Farm. Zh., 18 (1984) 114.

42 E. Lukevics, L. Ignatovich, N. Porsiurova and S. Germane, Appl. Organomet. Chem., 2 (1988) 115.

43 E. Lukevics and S. Germane, L. Ignatovich, Appl. Organomet. Chem., 6 (1992) 543.

44 Proxigermanium (SK-818), Drugs Fut., 18 (1993) 905.

Received: January 4, 1999 - Accepted: January 25, 1999 Received in revised camera-ready format: February 5, 1999 